\title{
Research of Multi-export Campus Network Upgrade Program Based on Redundant Architecture
}

\author{
Shan Jing \\ Shandong Provincial Key Laboratory of Network Based \\ Intelligent Computing \\ University of Jinan ,Jinan, China \\ Email: jingshan@ujn.edu.cn \\ Qi Qi \\ School of Information Science and Engineering \\ University of Jinan \\ Jinan, China \\ Email: ise_qiqi@163.com
}

\author{
$\mathrm{Na}$ Yang \\ School of Information Science and Engineering \\ University of Jinan \\ Jinan, China \\ Email: ise_yangna@163.com
}

\author{
Runyuan Sun \\ Shandong Provincial Key Laboratory of Network Based \\ Intelligent Computing \\ University of Jinan, Jinan, China \\ Email: sunry@ujn.edu.cn \\ Corresponding author
}

\begin{abstract}
In order to improve the stability and effectiveness when campus network user access to Internet, the paper, relied on the multi-export campus network environment and based on the analysis on characteristics and demands of the campus network users, completes the design of network upgrade program by adopting the proxy, firewall, routing policy and policy based routing. And it demonstrates the feasibility of the program by combining with application deployment in University of Jinan.
\end{abstract}

Keywords: campus network; redundancy; multi-export; proxy; firewall; policy based routing

\section{INTRODUCTION}

With the improvement of levels and the enlargement of scales in university information construction, there is a higher requirement of network service and network stability on campus. In China, the education network is almost used as the main export in the initial campus network construction. But with the kind of campus network users increasing and the demand of access to network resource growing, many colleges and universities use education network as well as other ISP recently [1-2]. Using multi-export provides a richer set of network resources for college students and teachers. At the same time, it puts forward higher requirements to network management, for example, how to build a secure and stable campus network? How to take advantage of network export to distribute the network traffic? How to improve the performance of campus backbone network? During the design and upgrade of network, university network administrators are required to study deeply these issues and related technologies and make rational deployment.

\section{SitUATION ANALYSIS}

Unlike general network, campus network, with multiple internal resources, large user groups, kinds of users and other characteristics, is more complex. With the improvement of university information system and the growth of the campus network utilization, there is higher demand for effectiveness and stability of campus network.

University of Jinan campus network was founded in 1996 that is the first one to officially have access to CERNET in Shandong Province, now has hundreds of information service platform. University of Jinan campus network has three IPv4 network exports: CERNET, China Unicom and China Telecom. CERNET and China Unicom, the main export links of campus network, each has an export bandwidth up to $1 \mathrm{Gbps}$.And China Telecom as an alternative export link of the campus network has an export bandwidth up to $100 \mathrm{Mbps}$.

IP addresses of the campus network user terminals mainly include education network address and the internal address. Users using education network address can access education network directly, but the users using internal address have only access to the campus network by default [3]. China Unicom and China Telecom also provide small IP address blocks. Administrators assign this part to public resource servers, proxy servers and equipment of network exports. As the default route of campus network points to CERNET exports, users need to use HTTP proxy while accessing Internet resources [4]. If the number of users is large, the efficiency is severely affected because the forwarding way that HTTP proxy server adopts is software. Meanwhile, campus network takes a long time to recover from network failures because it lacks effective redundancy design, the working and learning efficiency of campus network users could be affected to a certain extent. The following by combining with campus network upgrade work of University of Jinan studies deeply the program that adopts agent technology, firewall technology, routing policy and policy based routing. 


\section{Key TECHNOLOGY RESEARCH}

\section{A. Proxy Technology}

Proxy technology is a solution based on bypass mechanism to access the external network. Proxy server simultaneously accesses internal network as well as external network, and its default route points to the external network. When using a proxy server, a user needs to set the predefined proxy server address in the browser, target site information will be sent to the proxy server by the browser, then the proxy server establishes connection with the target site to get the information and send it back to the browser. Based on the above way, when the proxy server's buffer has corresponding information, it can directly set the corresponding data back to the browser to accelerate accessing speed of users. Meanwhile, the proxy server can also implement network security filtration, network traffic control, user management and other functions.

\section{B. Firewall Technology}

Firewall is a protective barrier between the internal network and external network and it is in the border of the network. Firewall technology can control communication between the internal network and the external network and prohibit an insecure network connection and increase the security of the internal network [5]. NAT is a key of firewall technology that can form mapping relationship between public address and private address and solve the problem of IP address shortage, and it is able to form isolation between internal network and external network and improve the security. The user can normally connect to the Internet without extra settings because NAT technology is transparent to users.

\section{Policy Based Routing}

The traditional route is based on the destination address of IP packet in the forwarding of packets. The policy based routing is a more flexible forwarding mechanism. The groups could select a different path according to the source address, protocol type, or application type by using policy based routing[6].If the IP packets and the relevant strategies of policy based routing match, data packet will be forwarded according to the specific next hop in policy based routing. If the match fails, data packet will be forwarded according to the traditional routing based on destination address. Policy based routing can be used to provide a more flexible routing approach for multi-export campus network.

\section{CAMPUS NETWORK UPGRADE PROGRAM}

The previous campus network export way of University of Jinan is HTTP proxy. It is a single way and is low in terms of work efficiency and stability. Meanwhile, the default route of campus network points to the CERNET export. After analyzing on campus network traffic, it found that most of resources accessed by the current campus network users distributed on the Internet, and the export traffic of education network was less than Internet. It results in excessive pressure on the proxy server. The upgrade program changes default route of the campus network to point to ISP-
ChinaUnicom after full consideration of these issues, at the same time, it adopts backbone redundancy strategy, redundancy strategy for export, and data distribution based on policy based routing and other measures to enhance the stability and effectiveness of the campus network. The Fig.1 shows the campus network update program topology.

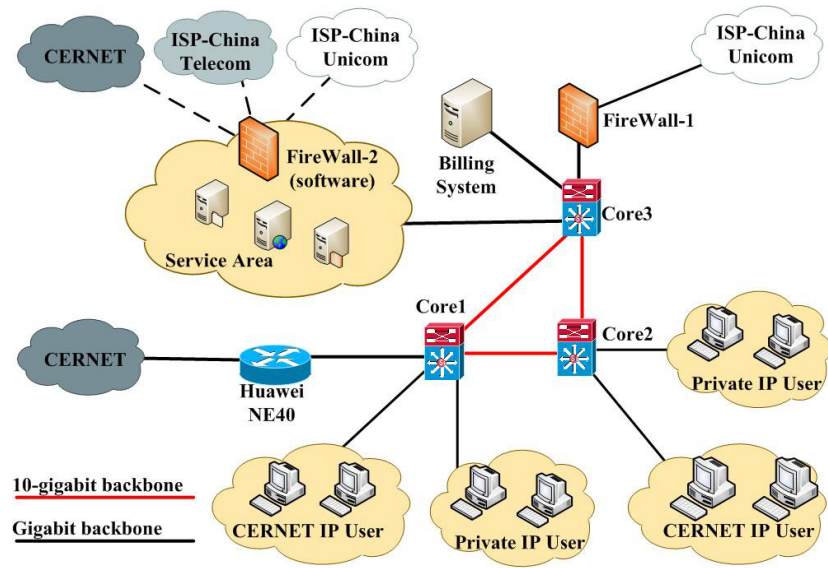

Figure 1. Campus network upgrade program topology

\section{A. Backbone Redundancy Strategy}

As the existing equipment Core 1 has been running for a long time, taking into account of reducing the operating pressure of Core1 and increasing backbone network redundancy, the upgrade program purchases core equipment Core 3 and forms a redundant loop in backbone network including Core1, Core 2 and Core 3 , it also migrates the link between the service area and Corel to connect the service area with Core 3 . Three core devices are built into a core ring network to increase the redundancy of link and improve the reliability of network. Using dynamic routing protocol in network can keep acyclic forwarding path acyclic and maintain backup link, also static routing policy could be used in network. Static routing can reduce occupation of equipment and bandwidth during routing update, so the program chooses a solution of static routing to achieve redundancy by adjusting the priority of alternative static routing entry. Core 1 is used to be an example to set static routing as follows:

$$
\text { ip route 0.0.0.0 0.0.0.0 Nexthop:Core3 }
$$

ip route 0.0.0.0 0.0.0.0 Nexthop:Core2 10

$$
\text { ip route Core2_IP_Networks Nexthop:Core2 }
$$

ip route Core2_IP_Networks Nexthop:Core3 10

The default route of Core 1 points to Core 3, it can step into the routing table and play a role. In the meanwhile the network administrator sets up redundant route to point to Core2. When the physical failure of the link between Core1 and Core 3 occurs, it could automatically switch to an alternative route and form the link of Core1-Core2-Core3. 
Similarly, for the network connected to Core2, make the next hop of Core1's static routing point to Core2. When the physical failure of link between Core1 and Core2 occurs, it could automatically switch to an alternative route and form the link of Core1-Core3-Core2.

\section{B. Redundancy Strategy for Export}

The users connect to the Internet through NAT technology after campus network upgraded, HTTP proxy server would exist as a redundant connection. Under normal circumstances, the default route of the entire campus network points to FireWall-1, the FireWall-1 changes address of education network and private network into public routable address assigned by ISP-ChinaUnicom through NAT technology and then send it to Internet. When network outages occur caused by failure on FireWall-1, campus network users can use proxy server temporarily to access external networks by setting the proxy mode. It can avoid the campus network paralysis caused by individual equipment failure to some extent and enhance the stability and availability of campus network. Meanwhile, the proxy server sets up proxy services aiming at different ISP. If resources accessed by the user are not on the default ISP, users can set appropriate proxy to achieve the interconnection. This architecture provides a more flexible access when the new ISP is added into campus network.

\section{Data Distribution Based on Policy Based Routing}

As the export bandwidth of campus network is limited, a lot of network traffic flow to a single export could increase the pressure of export equipment and reduce the availability of the campus network, it is necessary to implement data distribution policy based on policy based routing taking into account the different needs of campus network resources for different users.

The current major exports for the campus network are CERNET and ISP-ChinaUnicom, while the internal user's IP address includes education network address and the internal private address. If the user whose IP address is education network address accesses the education network resources, it can choose a way through Core1 to Huawei-NE40 which is border device of CERNET to achieve traffic distribution of education network. The packet whose source address and destination address are education network address could point to Huawei-NE40 by setting the appropriate policy based routing on the core device, the other data that does not comply with the policy points to FireWall-1. Taking into account that the user device may also access to Core 3 in the future, it needs to complete the appropriate policy based routing configuration in advance.

The following is critical configuration information of Core1:

access-list 101 permit IP Source_IP_UJN_CERNET

Destination_IP_CERNET

route-māp $\overline{d e f a u l t-r o u t e ~ p e r m i t ~} 10$

match ip address 101 set ip default next-hop IP_NE40

Policy based routing is used on CERNET network interface.

Configurations of Core2 and Core 3 are similar to Core1's, not repeat them here.

After the implementation of the above policy, the packet will take the default route when education network users access Internet resources and it will be sent to ISPChinaUnicom after the address translation performed by FireWall-1. It will match the policy based routing and be sent to CERNET by Huawei-NE40 when education network users access education network. Users of private network access education network resources by setting education network proxy.

\section{CONCLUSION}

The paper, relying on campus network deployment of University of Jinan, is designed and deployed for multiexport campus network redundancy program and has some practical significance. The program, integrated policy based routing, proxy technology and firewall technology, optimizes the campus network export strategy and increases export redundancy and backbone network redundancy to achieve campus network data distribution and to enhance the stability and effectiveness of the campus network. Successful implementation of the program can provide effective references for other campus network.

\section{ACKNOWLEDGMENT}

This work was supported by the Program for sub-project of the National Sci-Tech Support Plan (2012BAF12B07-3), and the next generation of Internet technology research and development, industrialization and large scale commercial projects (CNGI-12-03-017).

\section{REFERENCES}

[1] Zhang Zhicheng, ZOU Renming, ZHANG Xiaoquan and ZHU Junjun, "Design and Realization of Multi-Export Campus Network Based on Redundancy Architecture," Computer Science, Vol.39, No.10 Sup, pp.219-222, October 2012.

[2] YU Jiaming, ZHU Jinning, LI Zhilan, "Research of the Multi-Entry Campus Network Architecture," Computer Engineering \& Science, Vol.28, No.3, pp. 139-142, March 2006

[3] Y. Rekhter, B. Moskowitz, D. Karrenberg, G. J. de Groot and E. Lear, "Address Allocation for Private Internets," RFC 1918, February 1996.

[4] Kun Lei, Zhao Jinrong, "Network Security and the Proxy," Computer Engineering and Applications, No.14, pp.58-31, July 2001.

[5] PAN Wenchan, DONG Aihua, "LIU Shangdong. Research on Application of Network Security Based on Firewall Policy Routing," COMPUTER TECHNOLOGY AND DEVELOPMENT, Vol.23, No.8, pp.125-128, August 2013.

[6] YANG Lin, "Multi-output Configuration Based on Policy Routing in the Campus," Communications Technology, Vol.43, No.06, pp.123125, June 2010. 